Projets

de paysage

\section{Projets de paysage}

Revue scientifique sur la conception et l'aménagement de l'espace

$8 \mid 2012$

Les concepteurs de jardins et de parcs japonais

\title{
Le jardin comme dispositif de vision du temps
}

The garden as a time viewing device

\section{Aline Gheysens}

\section{OpenEdition \\ Journals}

Édition électronique

URL : http://journals.openedition.org/paysage/15649

DOI : $10.4000 /$ paysage. 15649

ISSN : 1969-6124

\section{Éditeur :}

École nationale supérieure du paysage de Versailles-Marseille, Institut national des sciences appliquées Centre Val de Loire - École de la nature et du paysage, École nationale supérieure d'architecture et de paysage de Bordeaux, École nationale supérieure d'architecture et de paysage de Lille, Agrocampus Angers

\section{Référence électronique}

Aline Gheysens, « Le jardin comme dispositif de vision du temps », Projets de paysage [En ligne], 8 2012, mis en ligne le 18 octobre 2012, consulté le 11 mars 2021. URL : http://

journals.openedition.org/paysage/15649; DOI : https://doi.org/10.4000/paysage.15649

Ce document a été généré automatiquement le 11 mars 2021.

Projets de paysage 


\section{Le jardin comme dispositif de vision du temps}

The garden as a time viewing device

Aline Gheysens

\section{Introduction}

1 Certains jardins ont la propriété de rendre perplexes : une fois saisis par eux, nous ne pouvons échapper au mystère de leur existence, des raisons de leur apparence actuelle, des multiples états qui ont précédé et succéderont à cette actualité, de leur improbable pérennité.

2 Mais est-ce à dire pour autant que le jardin, dans un génie insaisissable, se suffit à soulever de si puissantes réactions chez son visiteur? N'est-il pas plus vraisemblable que cette perplexité aille de pair avec toute interrogation mettant en jeu le temps de l'existence humaine ? Que le génie du jardin consiste plus précisément à colorer cette réaction de nuances agréables à l'esprit, rendant la conscience du temps non seulement supportable, mais même désirable? Le jardin, en somme, se présenterait comme un lieu à la fois vaste et accessible où inscrire par effleurements notre présence au monde ; comme une pratique autobiographique visant à nous mesurer, par le biais d'une expérience de beauté absolue ${ }^{1}$, à l'iridescence du temps, soit à sa propension à changer d'apparence en fonction de l'angle selon lequel on l'observe.

Puisque nous échouons à appréhender le temps dans la totalité de ses aspects et qu'il faut se limiter à le saisir de manière fragmentaire, je m'attacherai tout d'abord, pour le cas de La Petite Escalère, à identifier et questionner un ensemble de figurations du temps apparaissant au travers du récit afférent à la création, puis dans le rapport de simultanéité qu'il manifeste entre projet et expérience. Dans un deuxième temps, je tenterai de mettre en évidence des fonctions rétrospective et prospective du jardin, telles qu'elles sont mobilisées lors de la promenade. 
Que le jardin puisse former une œuvre autobiographique a maintes fois été observé. Ce qui reste à clarifier, dans le cas de La Petite Escalère, se situe plus précisément dans les problèmes posés par la restitution de ce phénomène d'incorporation de l'expérience offerte par un jardin autobiographique dans un processus d'écriture autobiographique. $\mathrm{Si}$, comme un certain nombre d'indices le signalent, l'écrivain puise dans l'expérience de son jardin des éléments indispensables à l'élaboration de son œuvre écrite; si cet espace, en la modelant, contribue à la singularité de cette écriture, donc à la réussite du projet d'autobiographie de son auteur, alors il faut trouver une manière de rendre compte de cette expérience du jardin dans toute sa propension à déclencher une «méditation recyclable » sur l'existence. C'est en partant de cette idée que j'ai mené pendant deux ans un observatoire photographique rejouant le parcours du marchand d'art-collectionneur-écrivain dans son jardin.

\section{Brève histoire ou mythe fondateur de La Petite Escalère}

5 La Petite Escalère a ceci de particulier, pour un jardin privé, qu'elle abrite une cinquantaine de sculptures parmi lesquelles figurent des œuvres de Rodin, Bourdelle, Léger, Miro, Matta, Oteiza, Calder ou Goldsworthy.

6 Son histoire débute au début des années 1960, lorsque Paul Haim, marchand d'art, et son épouse Jeannette Leroy, artiste, font l'acquisition d'une ferme à Saint-Laurent-deGosse (Pays basque). Par le hasard d'une piscine construite sur le terrain d'un voisin, le couple se voit contraint de lui acheter des hectares de champs de maïs et de forêt $a$ priori inexploitables.

7 Dans le courant des années 1970, Paul Haim organise une importante exposition de sculptures au Japon. En remerciement, il se voit offrir l'opportunité d'acheter plusieurs œuvres qu'il entrepose dans son jardin, encore nu. Ainsi débute une collection qui ne cessera de s'accroître au fil des années.

8 Jeannette Leroy, sans se départir de son œil de peintre, s'initie quant à elle à planter massivement, mue par le désir de voir disparaître les sculptures dans la nature. Très vite, l'aide d'un jardinier se fait sentir et entre en scène Gilbert Carty, qui contribue à transformer cet espace sans qualité en une œuvre où fusionnent nature et sculptures, elles-mêmes parfois faites de nature.

Une première remarque concernant le temps en représentation dans le jardin peut déjà être faite ici : à travers les propos rapportés par les acteurs principaux de La Petite Escalère, apparait de manière récurrente la référence à un "temps substrat ", temps zéro du jardin décrit comme indécelable dans l'espace actuel, mais sur lequel semble pourtant reposer la possibilité même d'une histoire.

10 Le philosophe Jean-Paul Larthomas, dans un recueil consacré à la lecture des jardins dans la littérature ${ }^{2}$, cite Goethe: "Je garnis et j'arrange tous les coins négligés [du jardin] avec des mains amoureuses! Je plante des arbres comme les enfants d'Israël posaient des pierres, en témoignage... L'agriculture est une chose admirable parce qu'elle donne toujours une réponse nette, qu'on fasse bien ou qu'on fasse mal ». Ce à quoi le commentateur ajoute que: "La pratique du jardin impose d'être à la fois botaniste, peintre et philosophe. » C'est peut-être précisément sur cette base tripartite ici personnifiée par Paul, Jeannette et Gilbert, qu'est né le jardin de La Petite Escalère, 
ou plus exactement son mythe fondateur, qui parlerait d'une terre où, comme dans tout commencement, il est loisible de penser qu'avant nous, il n'y avait rien. Un « rien " qui s'apparie, à la croyance en une absence initiale de savoir sur le jardin et qui va probablement de pair, en réalité, avec une impossibilité temporaire d'appropriation physique de cet espace.

11 Deuxième remarque : l'étendue du jardin et l'impression de réussite qui s'en dégage pourraient être le signe d'une certaine ambition de la part de ses concepteurs. Cette réussite pourrait avoir nécessité une antériorité du projet (esquisse, phasage des travaux, plans, mise en forme) par rapport à la modalité temporelle particulière de l'expérience du paysage ${ }^{3}$. On apprend au contraire que La Petite Escalère s'est créée sur le mode du phénomène spontané, sans cause et sans contrôle.

\section{Fonction rétrospective et figure du premier jardin}

Certains jardins s'accordent à témoigner d'un désir, pour leurs propriétaires, de réunir la somme des connaissances d'une époque, d'une civilisation ou, plus modestement, de rassembler et de figurer rétrospectivement les parts éparses d'une existence individuelle lorsque, suite à des déplacements successifs, celle-là ne peut plus être perçue de manière unitaire. De même il apparaît, pour les proches du propriétaire de ce jardin, que sa réalisation ait été le meilleur moyen d'obtenir que son propriétaire, après avoir vécu une large part de son existence en déplacement, parvienne à s'enraciner quelque part. La Petite Escalère, sous cet angle, reproduit, bien qu'à l'échelle individuelle, le " premier jardin » auquel Gilles Clément fait référence en entamant sa Brève histoire du jardin :

«Le premier jardin de l'Histoire n'est pas celui des livres d'histoire, mais celui des peuples qui au cours des temps - quelle que soit l'époque - ont cessé leur activité nomade pour se fixer en un point de leur territoire ${ }^{4}$. »

Plus loin il rappelle que le premier jardin est un enclos, car il convient de protéger le bien précieux du jardin : légumes, fruits, fleurs, animaux, mais tout autant art de vivre :

«Ce qui, au fil du temps, ne cessera d'apparaître comme "le meilleur". C'est la façon

d'interpréter le meilleur qui, en fonction des modèles de civilisation, va déterminer

le style des jardins ${ }^{5}$."

Si, dans le cas de La Petite Escalère, il est concevable que les sculptures correspondent à ce qui, aux yeux des propriétaires du jardin, existe de meilleur, il est moins aisé de croire que leur exposition permanente aux intempéries et aux autres agents érosifs puisse faire office de protection. Comment interpréter, alors, ce geste incongru de la part d'un expert en art, d'exposer des œuvres aux pluies et aux vents, quand la connaissance de leur valeur inciterait au contraire à les mettre à l'abri, les sculptures n'ayant pas nécessairement été pensées comme sculptures de jardin ou pour jardin? Parce qu'il est à comprendre - Jeannette le confirmera plus tard - comme un geste partant d'une impulsion et qui se soucie peu de la crainte de la perte. Pour saisir mieux cette contradiction, sans doute s'impose-t-il d'apprécier cet espace non plus en tant que socle ou écrin vivant pour objets précieux, mais plutôt pour ce que ces objets laissent apparaître comme traces d'une suite de mouvements opérés dans l'espace à travers le temps.

Peut-être devons-nous également lire David Lowenthal qui, dans son essai de géographie et d'histoire des représentations du paysage, met l'accent sur l'importance, 
pour les individus, de s'entourer de preuves conscientes et particulières du passé afin de s'enraciner dans un lieu. Faute d'une histoire intime et vivante, dit-il, nous avons besoin de souvenirs tangibles de ce que nous avons fait, vu, visité :

« $\mathrm{Si}$ nous vivions avec notre passé et notre présent, nos objets d'hier et d'aujourd'hui, nous nous sentirions toujours chez nous. Quand cela n'est pas possible, nous nous rabattons sur des souvenirs ou des mémentos qui remplacent des paysages évanouis ${ }^{6}$. »

Les sculptures, indépendamment du sens qu'elles peuvent contenir en elles-mêmes, en ravivant des pans perdus de l'existence du marchand d'art, participeraient donc à un dispositif mis en place par lui afin de lutter contre une certaine entropie qui corrode les œuvres mais aussi l'esprit de l'homme. En somme, s'il est quelque chose d'essentiel contre quoi ce jardin semble devoir protéger, c'est donc de l'oubli.

\section{Temps de la promenade}

17 C'est sur la base de ce postulat d'un jardin-mémento que je souhaite appréhender La Petite Escalère, par le biais d'une archéologie faisant appel, par montage, autant au vécu qu'au lu, au visible et palpable qu'à l'imaginaire jaillissant sous des apparences variées chez ses propriétaires, à leurs souvenirs vrais comme à leurs souvenirs fictifs, à tout ce qui n'attend, pour se déployer, que l'occasion d'une promenade. Parmi mes «matériaux» figure aussi le tour du jardin mené quotidiennement et suivant un déroulement invariable par Paul Haim en préalable à son activité d'écriture ${ }^{7}$. Car le collectionneur et marchand d'art est aussi l'auteur de romans et nouvelles à caractère autobiographique et d'essais relatifs à l'art ${ }^{8}$. Or, l'élaboration de son œuvre écrite coïncide temporellement avec celle du jardin. Questions sous-jacentes, dès lors : que fait faisait Paul Haim, quand il marchait? Quel est le sens de l'immuabilité de son parcours? Serait-il juste et opportun de l'assimiler, relativement à l'hypothèse d'une autobiographie paysagère, à un acte de lecture-relecture ? Si oui, le sens attribué à ce qui est donné à lire dans l'espace lors de la marche agit-il en vue d'une fixation de l'identité de l'écrivain ou bien, au contraire, en vue de la maintenir mouvante?

Son parcours, schématiquement, pourrait être caractérisé par la traversée de trois espaces distincts (qu'il conviendra de caractériser avec précision dans une phase ultérieure de recherche: orientations, prédominance d'ombre, de soleil, modalités du cheminement, présence de l'horizon, de l'extérieur du jardin, etc.), chaque espace donnant lieu pour le promeneur à la distinction de temps dont la teneur et les qualités diffèrent nettement les uns des autres.

Première séquence : espace jouxtant la propriété, rigoureusement quadrillé, objet de soins permanents, rappelant en cela le caractère rassurant du foyer.

Puis, dans un deuxième cercle concentrique partant du foyer, une région plus hostile de la forêt où les sculptures, éparses, ne sont pas données à voir d'emblée, mais dont la présence permet au visiteur d'organiser l'espace qui, sans cela, resterait brouillé, chaotique.

21 Enfin, aboutissement au lieu-dit du Moura, paysage décharné, sujet aux vents violents, expérience d'une durée pure, semblable à ce que peut connaître celui qui écrit, présent au monde et comme isolé de son bruit. 
C'est donc à mesure que le paysage se dénude que le visiteur apprend à voir. Le presque rien du Moura : un sol et un ciel et quelques brindilles, parce qu'il succède, dans l'ordre de la promenade, à une suite d'expériences esthétiques tantôt grandiloquentes, tantôt silencieuses, parce qu'il porte encore l'empreinte de chacune des sensations provoquées par ces expériences, revêt pour la vue un caractère extraordinaire. À plusieurs reprises, le paysage s'est transformé et la qualité du regard du promeneur avec lui. Parvenue à un point d'aboutissement, où son étendue est maximale, la vision devient possiblement contemplation.

\section{Un observatoire photographique}

23 Toute tentative de restitution de cette expérience, en ce qu'elle comporte d'intraduisible, se heurte fatalement au risque d'un échec. C'est pourquoi j'ai mis en place depuis près de deux ans un observatoire photographique à La Petite Escalère consistant à rejouer la promenade de Paul Haim à différents moments du jour et de l'année. L'enjeu de cette opération est à la fois d'atteindre une saisie plus fine de ce jardin, de documenter l'infinité de variations auxquelles il est sujet, mais aussi de rendre compte, par la spécificité d'une pensée par l'image, de son interrelation avec l'œuvre autobiographique écrite du collectionneur.

Le temps de la conception de La Petite Escalère correspond, pour chacun des membres du couple Haim-Leroy, au moment particulier de l'existence où l'horizon temporel bascule $^{9}$, où un renversement s'opère entre la place donnée au possible à venir et le passé à remémorer ; où un réajustement s'impose par rapport à une image de soi et du temps qu'il reste à vivre. $\mathrm{Si}$, comme le souligne $\mathrm{Yu} \mathrm{Fu}$-Tuan, "l'espace et le temps coexistent, s'entremêlent et se définissent l'un l'autre dans l'expérience temporelle ${ }^{10}$ ", cela signifie qu'il est possible de trouver, dans l'espace et l'histoire de La Petite Escalère, des traces manifestes de telles opérations de réajustement. Celles-là se décèlent dans une sensation d'étrangeté temporelle, de brouillage. Impossible en effet de définir, pour le visiteur, un point précis dans cet espace où tout aurait commencé, où se situe la plus récente intervention ; tout semble avoir toujours été là. Ou plutôt, être apparu cette nuit et en une seule fois.

En cela, il en va de ce jardin comme du rêve où, selon le psychanalyste André Green, «tout est d'un pur présent »; le jardin, comme le rêve, semble démontrer l'existence d'un temps "éclaté ", "démembré ", "non unifié », auquel la conscience diurne ne donne quasiment jamais accès, à l'exception de «moments de "temps sensibles" (Julia Kristeva), “qui réapparaissent inopinément, par bouffées si enivrantes qu'on risque de perdre connaissance" $" 1$ ".

26 Pour le visiteur, le trouble est double: car cette impression de "pur présent» s'évanouit si son regard se heurte à l'aspect de certaines œuvres, dont l'usure de la surface ne peut dissimuler l'effet et la présence du temps. À l'érosion subie se superpose aussi un phénomène de brouillage lié à la temporalité intrinsèque de chaque sculpture, qui se révèle à travers sa forme, ses matériaux constitutifs; tout ce qui se situe en hors-champ, tout le monde qu'elle convoque imaginairement, dans l'esprit du visiteur, avec la fulgurance d'un météore. C'est dans cet interstice que l'approche photographique proposée plus haut peut apporter sa pierre : car elle se situe en deçà du seuil de la restauration par la raison, gelant artificiellement la sensation de temps alors que la confusion y règne encore en maître. Un « hors temps » et « tous les temps » $y$ 
culminent dans un état innommable : elle peut tendre à montrer quelque chose du paysage tel qu'il ne peut être dit.

D'une certaine manière, le trouble opérant à La Petite Escalère ressemble à ce que Marc Augé désigne sous l'expression de "temps pur», temps sans histoire dont seul l'individu peut prendre conscience et dont le spectacle des ruines peut lui donner furtivement l'intuition ${ }^{12}$. Le paysage des ruines, selon l'anthropologue, a ceci de particulier qu'il ne reproduit intégralement aucun passé et fait intellectuellement allusion à de multiples passés, proposant au regard et à la conscience la double évidence d'une fonctionnalité perdue et d'une actualité massive, mais gratuite.

En début de ce texte, la question posée était celle des dispositifs mis en œuvre au sein du jardin afin de faire apparaître à son visiteur un rapport entre le temps de son existence et d'autres phénomènes temporels rendus accessibles à sa conscience. De ce qui a été dit jusqu'ici je peux conclure qu'au moins deux phénomènes se produisent successivement et de manière quasi subliminale, lorsqu'on déambule dans ce jardin : l'un, en estompant les différentes étapes de sa réalisation, provoque une sensation de " pur présent ", permettant au visiteur de s'unir pleinement au lieu, de faire corps avec ce qui l'entoure. Tandis que l'autre invite le visiteur à quitter imaginairement le temps présent et à diverger vers tous les temps passés, futurs et même non advenus.

Notons que la ruine à laquelle fait référence Marc Augé ne se résume pas à ce qui reste, ravagé après destruction; une seconde acception correspondant assez justement au spectacle qu'offre La Petite Escalère est à envisager, comme un "chantier où quelque chose s'édifie par blocs de temps "; elle «n'est pas le résultat d'une soustraction, mais d'un ensemble de formes inédites et évolutives qui ne cessent de se métamorphoser dans le regard de qui s'y attarde [...]». On comprend, suivant ce raisonnement, le curieux tour de passe-passe à l'œuvre dans les dispositifs paysagers de La Petite Escalère, où ce qui, communément, s'associe aux vestiges, au temps ancien, à savoir la sculpture, n'est pas uniquement ce qui assimile le jardin à un "paysage de ruines "; cette assimilation est tout autant provoquée par ce qui ne cesse de s'y métamorphoser suivant des rythmes variables: végétation, humidité, luminosité, température. Ces variations, en cette contrée, sont si rapides qu'elles prennent un tour inquiétant: les chemins encore nettement dessinés hier, immergés aujourd'hui, affichent une impraticabilité absolue ; la prêle en quantité innombrable est venue s'installer à travers l'eau, mais comment? Et après, quand le soleil baignera à nouveau les sentiers, ne laissant de trace de l'inondation qu'un aspect luisant à la surface de la terre, comment rester convaincu de la véracité de souvenirs pourtant vivaces?

Il me semble utile, à ce stade de l'exploration, d'examiner le second matériau constitutif de l'archéologie que j'entends mener à La Petite Escalère, à savoir les écrits de Paul Haim. Puisque l'écriture accompagne de manière très serrée sa fréquentation du jardin, il m'est permis de penser que l'un et l'autre espaces élaborés par lui ont pu donner lieu à des phénomènes d'échanges et de transformations. Un peu à la manière dont le jardinier philosophe Jorn de Précy ${ }^{13}$ a pu le faire pour lui-même, livrant dans un essai la somme des jardins qu'il aima le plus et leur influence sur celui qu'il réalisa luimême. Il s'agit de constituer, notamment au moyen de photos prises par le couple dans leur jardin depuis ses débuts ou lors de visites de jardins - au Japon notamment-, la biographie jardinière et paysagère de Paul Haim.

31 Certains motifs, certaines figures apparaissent de manière récurrente d'un écrit à l'autre de Paul Haim. «Qui tente de s'approcher de son propre passé enseveli doit faire 
comme un homme qui fouille", dit Walter Benjamin ${ }^{14}$. «Il ne doit surtout pas craindre de revenir sans cesse à un même et seul état de choses - à le disperser comme on disperse de la terre, à le retourner comme on retourne le royaume de la terre car les "états de choses" ne sont rien de plus que des couches qui ne livrent qu'après une exploration méticuleuse ce qui justifie ces fouilles. [...] Au sens le plus strict, le véritable souvenir doit donc, sur un mode épique, rhapsodique, donner en même temps une image de celui qui se souvient, de même qu'un bon rapport archéologique ne doit pas seulement indiquer les couches d'où proviennent les découvertes mais aussi et surtout celles qu'il a fallu traverser auparavant. »

Si son second roman s'ouvre comme la suite du premier, il n'empêche que le troisième entrechoque les souvenirs et confond un lecteur en attente de confort chronologique. De même que des sauts s'opèrent entre paysages connus, perdus et à conquérir ${ }^{15}$ pour que se tisse une géographie subjective où se condensent paysages contemplés et paysages remémorés par eux :

« Aujourd'hui je regarde ma main boucanée tracer ces lignes. Des décennies se sont écoulées. Je lève les yeux et j'entrevois de mon fenestron les reflets de l'Adour dansant dans la lumière de l'automne. J'ai autant de souvenirs, comme disait Baudelaire, que si j'avais vécu mille ans. Je me souviens du majestueux plan d'eau que nous traversions de Brazza à Léo. Le Stanley Pool. Plan d'eau de vingt kilomètres d'envergure séparant deux populations francophones dissemblables ${ }^{16}$. "

Si un paysage, dans les écrits de Paul Haim, apparaît parfois comme ce qui demeure d'une proximité perdue ${ }^{17}$ ou ce qui provoque une proximité rêvée avec des êtres chers $^{18}$, il est bien souvent imbriqué dans un système complexe de relations où fourmillent les évocations à d'autres paysages, lieux, jardins, eux-mêmes associés à des œuvres ou à des artistes, voire à des œuvres présentes à La Petite Escalère. Le recueil de nouvelles Tel un fil de pourpre abonde d'exemples illustrant ce cas de figure. La forêt du Douanier Rousseau par exemple, « extrapolation de la jungle » s'associe dans l'esprit de l'auteur au zoo de Vincennes en même temps qu'au souvenir de son incursion aventureuse et hallucinée dans la forêt équatoriale (Haim, 1991, p. 58).

C'est certainement en procédant par montage de ces différents éléments que peut émerger une vision adaptée à la morphologie particulière de La Petite Escalère, nourrie d'une connaissance précise du rapport unissant le collectionneur-écrivain aux œuvres et à leurs temporalités. Les romans de Paul Haim fournissent également de nombreux indices concernant ses expériences de rencontres décisives avec des œuvres en les incorporant dans le temps de son existence : «Je n'avais jamais visité une exposition. Je ne savais rien de Rodin. "La Cathédrale" m'avait bouleversé. Je pressentais que la sculpture pouvait me procurer des émotions fortes, enrichissantes.» (1991, p. 175). D'autres exemples nous renseignent sur son rapport aux œuvres, corrélées au temps dans une acception plus large : aux poteries de Picasso, il voue un culte par écrit, car s'inspirant des formes primordiales des civilisations méditerranéennes, évoquant la Grèce, l'Espagne et l'art hispano-américain, elles sont déjà de tous les temps (1995, p. 188). En ceci Paul Haim témoigne d'une posture présente chez des historiens de l'art tels que Georges Didi-Huberman, qui consiste à étudier la flexibilité temporelle des œuvres, leur capacité à être à la fois très modernes et très anciennes, à opérer des glissements du passé dans le présent ${ }^{19}$. 


\section{Exploration du « hors-champ » des sculptures}

L'étude du " hors-champ » des sculptures fait apparaître une œuvre comme Les Femmes au perroquet de Fernand Léger sous un jour nouveau. La biographie de l'artiste nous apprend que la représentation de figures flottantes et leurs fonds abstraits organisés en bandes parallèles de couleurs primaires s'associe à son expérience de l'environnement urbain américain et plus exactement au choc visuel produit par les publicités lumineuses de Broadway ${ }^{20}$. Il est probable que cette convocation de New York dans la fresque de Léger n'ait pas échappé à Paul Haim, puisqu'il affiche dans ses romans une connaissance à la fois experte et intime de son " maître ». Relatant leur rencontre, il y évoque (ou plus exactement met en scène une évocation provenant non de cette rencontre, mais d'un travail de documentation postérieur à celle-ci) le souvenir qu'a Léger d'une ferme louée à la frontière canadienne lors d'un été caniculaire et dont l'état d'abandon aurait frappé le peintre. Alors que dans sa Normandie natale, les paysans utilisaient chaque branche morte, de même qu'ils récupéraient chaque $\mathrm{clou}^{21}$, là, au bout d'un champ où les ronces avaient enfoui des charrues, des herses, des roues de carriole, « la nature reprenait ses droits sur la technique » (Haim, 1995, p. 252). Le contraste entre la végétation et des objets mécaniques, l'usure accélérée et la rapidité de renouvellement des biens de consommation étaient perçus par Léger comme un signe de dynamisme et de vitalité. On retrouve des traces de ces paysages dans des œuvres ultérieures telles que Adieu New York (1946, Centre Pompidou). On ne peut, partant de là, prétendre que le choix, pour Paul Haim, d'implanter la fresque de Léger en lisière de forêt plutôt que sur une pelouse nette ait un rapport clair avec le souvenir de Léger de la ferme abandonnée. Mais on peut en revanche présager que chaque œuvre, soumise à l'examen de son orientation et de son positionnement dans le jardin, des végétaux dont elle s'accompagne, la fera apparaître dans un système d'emboîtements multiples propice au déploiement d'un regard informé sur le jardin.

\section{Des systèmes d'emboîtements}

Il arrive également que l'écrivain disperse dans ses récits une allusion à une œuvre littéraire qui, une fois réintégrée au contexte de l'élaboration du jardin, produit une vision nouvelle du lieu. Si l'on ne peut prouver que Paul Haim ait transfiguré et déplacé des fragments de textes importés tels quels de ses lectures vers l'espace de son jardin, on ne peut douter que ce qui constitue le fonds de son imaginaire et jaillit dans les écrits y ait laissé des traces. Alors le jardin cesse d'être "espace clos ", s'ouvre et peut donner le vertige. De cela il faudra traiter avec soin ; tout au plus ici puis-je fournir au lecteur de minuscules prélèvements : de la description de sa rencontre avec l'écrivain Alejo Carpentier (Haim, 1995, p. 324), aller vers Los Pasos Perdidos, dont Haim dit garder le souvenir d'une lecture laborieuse et du foisonnement de descriptions de végétations majestueuses (ibid.,p. 325). Dans ce roman autobiographique, le protagoniste quitte épouse et maîtresse pour aller à la rencontre d'une femme primitive et d'un paysage perdu entre chaos et cosmos, dont il inonde le lecteur d'images exubérantes :

«Là, derrière les derniers toits, se dressaient les premiers arbres de la forêt vierge encore éloignée, ses postes avancés, ses superbes sentinelles, ressemblant à des obélisques plus qu'à des arbres, disséminés encore, distants les uns des autres, sur la vaste étendue accidentée du maquis coupé de bois, dont la fertilité à ras du sol 
effaçait les sentiers en une nuit. Le cheval n'avait rien à faire dans un monde qui

n'avait plus de routes ${ }^{22}$. "

Imprégnée de la présence sensuelle de la nature convoquée dans ces descriptions, de retour à La Petite Escalère, il me faut requalifier ma vision.

\section{Conclusion}

Nous voici, au terme de cette première exploration de La Petite Escalère, face à un schéma triangulaire où s'unifient et se construisent expérience du temps vécu, expérience littéraire et expérience du jardin. La boucle est bouclée: l'homme, cheminant à travers son jardin, est lui-même traversé par le temps de la nature et des œuvres qui le transforment; de retour dans son foyer, impressionné encore par l'expérience de marche et de contemplation, invite au souvenir, à l'imagination, mais aussi à la présence à soi, il transpose le temps vécu en récit. Quitte ensuite son bureau, transforme son jardin.

D'un mouvement qui menait Paul Haim, dans la première phase de son existence, vers un monde immensément vaste, l'élaboration du jardin et avec elle son appropriation semblent indiquer la fin. S'ensuit, pour le marchand d'art devenu écrivain, une phase d'introspection, mouvement inverse, partant de l'immensité d'un monde vu, lu, vécu, et traversé. Mouvement utilisant, par l'exercice de la promenade, la force du jardin à déployer des temps passés, présents et non advenus dans un monde en miniature.

Si pour Paul Haim, parvenu au seuil de la vieillesse, le jardin, en tant qu'élaboration autobiographique, s'impose dans un rapport étroit avec une démarche d'écriture, c'est que l'expérience offerte par la promenade, immuable dans son parcours mais infinie dans la lecture qu'elle propose et ce vers quoi elle oriente l'attention de son auteur, lui propose un dispositif de lecture-écriture qui dirait quelque chose de lui dans un langage toujours juste et toujours neuf, jamais rigide. L'écriture de soi, lorsqu'elle passe par l'intermédiaire d'un jardin, opère par effacements et réinscriptions sur les mêmes lignes. Ce faisant, elle dépasse les limites de l'écriture en préservant la motilité de l'identité de l'auteur, quand la page la fixerait. En clair, il se pourrait qu'en construisant soigneusement l'une et l'autre œuvres autobiographiques, Paul Haim ait mis au point un dispositif lui assurant la pérennisation de son existence dans la mémoire collective sans avoir à en subir l'angoisse collatérale.

Cette version constitue le prolongement d'un texte présenté lors des journées doctorales de paysage, IVe édition, ENSP Versailles-Marseille, 24 et 25 novembre 2011. J'adresse mes remerciements à Catherine Chomarat-Ruiz, qui dirige ma thèse, ainsi qu'à Catherine Grout, pour leurs commentaires. Merci également à Dominique Haim, Jeannette Leroy et Gilbert Carty pour la chaleur de leur accueil lors de mes visites à La Petite Escalère. 


\section{BIBLIOGRAPHIE}

Augé, M., Le Temps en ruines, Paris, Galilée, 2003.

Barbe, J.-P., Pigeaud, J., Histoires de jardins. Lieux et imaginaire, Paris, PUF, 2001.

Benjamin, W., « Fouilles et souvenir, dans Images de pensée, Paris, Christian Bourgois, coll.

« Titres », 2011, p. 181-182.

Carpentier, A., Le Partage des eaux (1956), Paris, Gallimard, 1991 (Los Pasos Perdidos, 1953).

Clément, G., Une brève histoire du jardin, Paris, J.-C. Béhar, 2011.

Fraisse, P., Psychologie du temps, Paris, PUF, 1967.

Fu Tuan, Y., Espace et lieu, la perspective de l'expérience (1997), Gollion, Infolio, coll., « Archigraphy

Paysages », 2006.

Green, A., Le Temps éclaté, Paris, Minuit, 2000.

Haim, P., Tel un fil de pourpre : vingt-deux histoires vraies, Paris, L'Harmattan, 1996.

Haim, P., Marchand de couleurs, Paris, L’Harmattan, 1995.

Haim, P., Passage du désir, Paris, Éditions de la Différence, 1991.

Jacob, M., Paysage et temps, Gollion, Infolio, coll. « Archigraphy Paysages », 2007.

Lasalle, H., Léger, tout l'art, Paris, Flammarion, 1997.

Lowenthal, D., Passage du temps sur le paysage, Gollion, Infolio, 2008.

Précy, J. de, Le Jardin perdu, Arles, Actes Sud, 2011.

Rebecchini, G., « Temporalité de l'œuvre d'art et anachronisme », Perspective, 2010-2011-3,

p. 461-468.

Thiébaud, P., Dictionnaire des jardins et paysages, Paris, Jean-Michel Place, 2007.

Van Lier, H., Les Arts de l'espace, Tournai, Casterman, 1959, p. 70.

\section{NOTES}

1. Cette forme de beauté, distincte de la beauté relative (beauté d'agrément, plaisir attendu pour lui-même), se définit, selon Henri Van Lier, par « une sensibilité renonçant provisoirement à son plaisir et s'ouvrant activement à un système d'autant plus fécond qu'il est différent d'elle, et se prépare ainsi à ce plaisir objectivant que nous appelons joie ou béatitude. », cf. Van Lier, H., Les Arts de l'espace, Tournai, Casterman, 1959, p. 70.

2. Cf. Larthomas, J.-P., « Le jardin selon Shaftesbury : une origine possible du romantisme », dans Barbe, J.-P., Pigeaud, J., Histoires de jardins. Lieux et imaginaire, Paris, PUF, 2001, p. 203.

3. Cf. Jacob, M., Paysage et Temps, Gollion, Infolio, coll. « Archigraphy Paysages », 2007, p. 7.

4. Cf. Clément, G., Une brève histoire du jardin, Paris, J.-C. Béhar, 2011, p. 11.

5. Ibid., p. 12.

6. Cf. Lowenthal, D., Passage du temps sur le paysage, Gollion, Infolio, 2008, p. 162.

7. Lors de la rédaction de la thèse, on distinguera plus finement promenade individuelle, promenade accompagnée de proches et jours d'avènement de nouvelles sculptures rompant avec 
le temps du quotidien; chacune de ces modalités d'appréhension induisant une expérience esthétique particulière du jardin pour le marcheur.

8. Cf. Haim, P., Soledad et Montserrat, Paris, Phébus, 2005 ; Matta, Paris, Séguier, 2001 ; Le Roman de Guernica, Paris, Albin Michel, 1999; Tel un fil de pourpre: vingt-deux histoires vraies, Paris, L'Harmattan, 1996 ; Marchand de couleurs, Paris, L'Harmattan, 1995 ; Passage du désir, Paris, Éditions de la Différence, 1991 ; Michel Seuphor, une vie à angle droit, Paris, Éditions de la Différence, coll. « Une vie pour l'art », 1988.

9. Cf. Fraisse, P., Psychologie du temps, Paris, PUF, 1963, p 189-190: «Tout se passe comme si l'homme, en se situant dans le temps, compte tenu de l'espérance de vie moyenne, attachait la plus grande importance à la partie la plus longue de sa vie, c'est-à-dire celle qui n'est pas encore vécue quand il est jeune et celle qu'il a déjà vécue quand il est âgé. Ce point de vue permet sans doute d'expliquer qu'entre 40 et 50 ans, il y ait dans toute vie humaine une période critique où on passe progressivement de la jeunesse à la vieillesse avec tous les réajustements des perspectives temporelles que cette mutation entraîne. "

10. Cf. Fu Tuan, Y., Espace et Lieu, la perspective de l'expérience (1997), Gollion, Infolio, coll., « Archigraphy Paysages », 2006, p 133.

11. Green, A., Le Temps éclaté, Paris, Minuit, 2000, p. 51. L'auteur insiste par ailleurs sur la clarté de vision produite par l'absence de chronologie dans un de ses rêves : « Mais oui, je revoyais tout, rien n'avait changé, ma mère, dans le rêve portait la robe que j'aimais voir sur elle quand j'étais enfant, je me retrouvais sur les lieux de mes vacances de telle époque en sa compagnie, plusieurs années après le temps où elle portait la robe en question. "

12. Cf. Augé, M., Le Temps en ruine, Paris, Galilée, 2003, p. 38-39.

13. Cf. Précy, J. de, Le Jardin perdu, Arles, Actes Sud, 2011.

14. Benjamin, W., "Fouilles et souvenir", dans Images de pensée, Paris, Christian Bourgois, coll. « Titres », 2011, p. 181-182.

15. Ainsi Paul Haim, évoquant son passage à Lisbonne : «Cette ville est déjà sud-américaine » (1991, p. 226) ; ou encore, décrivant Sao Paulo : «J'étais à Madrid, Rome ou Milan, telles que je les imaginais » (1995, p. 126).

16. Haim, P., Tel un fil de pourpre. Vingt-deux histoires vraies, Paris, L'Harmattan, 1996, p. 44.

17. Alors que la guerre le sépare physiquement de sa famille, Paul Haim reconstitue le lien en procédant par condensation de lieux : «Oncle Mikail habitait un bel immeuble, à l'angle de deux rues derrière le palais d'Hiver, qui rappelait Versailles à ma mère. »(1991, p. 247).

18. C'est le cas de Roland Barthes, qu'il déplore de n'avoir pas connu, alors qu'ils auraient pu être voisins : «Je regarde ce soir tomber la nuit sur ce fleuve qui a été aussi le vôtre. » (1991, p. 201.)

19. Cf. Rebecchini, G., « Temporalité de l'œuvre d'art et anachronisme », Perspective, 2010/2011-3, p. 461-468.

20. Cf. Lasalle, H., Léger, tout l'art, Paris, Flammarion, 1997, p. 65.

21. Ibid., p. 66.

22. Cf. Carpentier, A., Le Partage des eaux (1956), Paris, Gallimard, 1991 p. 122.

\section{RÉSUMÉS}

Partant du cas de La Petite Escalère, jardin de sculptures situé au Pays basque, ce texte propose une étude exploratoire du jardin qui l'envisage en tant que dispositif spatial acheminant 
l'individu vers une expérience intime de vision du temps. En prenant appui sur diverses réflexions prenant pour objet espace et temps, elle fait apparaître, dans le cas du propriétaire de La Petite Escalère, qu'espace littéraire et espace du jardin, élaborés simultanément, sont conçus et agencés comme un système d'écriture autobiographique aux interconnexions multiples.

This paper proposes to consider the garden as a spatial system giving rise to an intimacy experience of vision and retranscription of time. That exploratory study is illustrated by the case of La Petite Escalère, a sculpture park located at the French Basque country. Using previous studies about space and time, it is shown that this spatial system can be compared with an autobiographical writing practice. That exploration relies on the fact that, in the case of the owner of the garden, literary and garden spaces, which were designed and layed out simultaneously, are deeply interconnected.

\section{INDEX}

Mots-clés : jardin, temps, sculpture, photographie, écriture autobiographique

Keywords : Garden, time, sculpture, photography, autobiographical writing

\section{AUTEUR}

\section{ALINE GHEYSENS}

Titulaire d'un master 2 théories et démarches du projet de paysage (École nationale supérieure du paysage de Versailles, 2010) et d'un master 2 arts plastiques, visuels et de l'espace (ERG, Bruxelles, 2009), Aline Gheysens est doctorante en $2^{\mathrm{e}}$ année au sein de l'École doctorale Abies et du Laboratoire de recherche de l'École du paysage de Versailles-Marseille (Larep), sous la direction de Catherine Chomarat-Ruiz. alinegheysens[at]gmail[dot]com 